Research Article

\title{
The Excretion of BRCAA Antibody-Conjugated Gold Nanoclusters from Kidneys of Treated Rats
}

\author{
Kunlu Liu ${ }^{1 \#}$, Haixia $\mathrm{Li}^{1 \#}$, Yajun $\mathrm{Wu}^{1}$, Chunlei Zhang ${ }^{2}$, Duanyun $\mathrm{Si}^{3}$, Quansheng $\mathrm{Li}^{3}$, Sa Zhang ${ }^{4}$ \\ Jiye Yin ${ }^{1}$, Yuanyuan Liu ${ }^{1}$, Wensheng $\mathrm{Qu}^{1}$, Junhua $\mathrm{Wu}^{1}$, Yingge Zhang ${ }^{1}$, Ruibin $\mathrm{Su}^{1}$, Yuxia Wang ${ }^{1}$ \\ ${ }^{1}$ State Key Laboratory of Toxicology and Medical Countermeasures, Institute of Pharmacology and Toxicology, Academy of Military \\ Medical Sciences, Beijing 100850, China. \\ ${ }^{2}$ Institute of Nano Biomedicine and Engineering, Key Laboratory for Thin Film and Microfabrication Technology of the Ministry \\ of Education, Department of Instrument Science and Engineering, School of Electronic Information and Electrical Engineering, \\ Shanghai Jiao Tong University, 800 Dongchuan Road, Shanghai 200240, China. \\ ${ }^{3}$ Tianjin State Key Laboratory of Pharmacokinetics and Pharmacodynamics, Tianjin Institute of Pharmaceutical Research, Tianjin \\ 300193, China. \\ ${ }^{4}$ National Center of Biomedical Analysis, Academy of Military Medical Sciences, Beijing 100850, China. \\ ${ }^{\#}$ Authors equally contributed to the work. \\ Corresponding authors. E-mail: wangyuxia1962@hotmail.com
}

Received: Mar. 15, 2017; Accepted: Mar. 17, 2017; Published: Mar. 24, 2017.

Citation: Kunlu Liu, Haixia Li, Yajun Wu, Chunlei Zhang, Duanyun Si, Quansheng Li, Sa Zhang, Jiye Yin, Yuanyuan Liu, Wensheng Qu, Junhua Wu, Yingge Zhang, Ruibin Su, and Yuxia Wang, The Excretion of BRCAA Antibody-Conjugated Gold Nanoclusters from Kidneys of Treated Rats Nano Biomed. Eng., 2017, 9(I):36-48.

DOI: $10.5101 /$ nbe.v9i1.p36-48.

\begin{abstract}
The gold nanoclusters (Au-NCs) have emerged for delivery of various payloads such as small molecular drug and big molecular proteins due to their low immunogenicity, quick clearance and good biocompatibility. Even though they have shown great prospective in biomedical area, there are also some security problems which should be investigated further more. BRCAA1 mAb Au-NCs is a probe for gastric cancer diagnosis prepared by conjugating BRCAA1 monoclonal antibody with fluorescent Au-NCs. In this research, the renal excretion and effect on glomerulus filtration of BRCAA1 mAb AuNCs in rats were investigated. The result indicated that BRCAA1 mAb Au-NCs could be eliminated through the glomerulus filtration as early as $2 \mathrm{hr}$ after the administration following the excretion of other proteins in the plasma such as immunoglobulin. Electron microscope image and pathological analysis of kidney showed that BRCAA1 mAb Au-NCs induced some structural injury such as capillaries endotheliocyte damage, pycnosis, karyorrhexis and hyalomitome acidophilia enhancement. Biochemical indexes including ALT, AST, BUN, TBIL, CK and CREA are abnormal in the period of 2 to $48 \mathrm{hr}$ after the administration. The results of Western blotting and immunohistochemisty show the downregulation of three key proteins (ZO-1, Nephrin and Podocin) indicating the influence of BRCAA1 mAb Au-NCs on the foot processes of glomerular epithelial cells and shallow intercellular junctions or slit diaphragms.
\end{abstract}

Keywords: Gold nanoclusters; BRCAA1 antibody; Renal excretion; Glomerular filtration

\section{Introduction}

Fluorescent metal nanomaterials are used in medical applications such as photothermal therapy, radiotherapy and radiosensitizers, which have generated massive interest among researchers due to their biocompatibility, superior photostability, nontoxicity and outstanding fluorescent properties [1-8]. Gold nanoclusters (Au-NCs) are a kind of materials typically consisting of several to tens of gold atoms 
with extremely small size at an average core diameter less than $2 \mathrm{~nm}$ [9-10]. Au-NCs have attracted more attention in the past decade because of their ultra small size, excellent luminescence properties and stable structures or good storage stability [11-14]. In the recent years, gold nanoparticles have emerged for delivery of various payloads into their targets [15-20]. The payloads include small molecule drugs and big molecular proteins such as antibody and peptide. The toxicity of gold nanoparticles in vivo is directly related to their size, shape [21-22], surface coating, exposure dose and administration routes [23]. Developing favourable nanomaterials with efficient renal clearance is extremely important to their in-vivo biomedical applications [24, 25]. It has been demonstrated that the size plays a dominant role [26]. The size of gold nanoparticles is usually over $10 \mathrm{~nm}$ of diameter. Even though they have shown great prospective in cancer diagnosis and therapy, their safety remains being worried for their accumulation forming largesize particles in liver and spleen and thus inducing the difficulty of metabolism or excreation [27]. Ideal nanomaterials should have little accumulation in organs, be cleared out of the body effectively and show minimum interference with other diagnostic tests [28]. So the advantage of Au-NCs is the small size of 2-nmdiameter which can penetrate kidney filtration barrier and guarantee to decrease in-vivo toxicity by renal clearance. In the past few years, Au-NCs have been applied to recognize, track or even kill early gastric cancer cells for early diagnosis and therapy of gastric cancer. Moreover, molecular imaging technologies based on multifunctional nanoprobes have made great progress. Therefore, Au-NCs kidney toxicity in vivo has attracted our attention.

In the previous work, we observed that BRCAA1 (breast cancer associated antigen 1) is over expressed in gastric cancer cell lines such as SGC-7901, MKN1, MKN-74, MGC803 and KATO-III. We screened out and cloned brcaa1 gene from breast cancer cell line MCF-7 cells (AF208045, also called ARID4B, ATrich interactive domain-containing protein $4 \mathrm{~B}$ ), and identified its epitope peptide of SSKKQKRSHK [29]. Then we prepared BRCAA1 monoclonal antibody and predicted that BRCAA1 protein might be one potential targeting molecule for in-vivo gastric cancer cells at early phase. Herein, we conjugated BRCAA1 monoclonal antibody (BRCAA1 mAb) with fluorescent Au-NCs as gastric cancer probe, BRCAA1 mAb Au-
NCs, for early diagnosis of patients with gastric cancer. The objective of this study was to assess the main clearance way of BRCAA1 mAb Au-NCs from rats and to search the toxicity following its metabolism by evaluating the biodistribution, biochemistry, hematology and histopathology.

\section{Materials and Methods Materials}

PageRuler ${ }^{\mathrm{TM}}$ Prestained Protein Ladder, \#26617, Lot: 00169269, was from Thermo Scientific. Rabbit Anti-GAPDH antibody [EPR16884] ab181603, and Rabbit Anti-Nephrin antibody [Y17-R] ab136894, were from Abcam. Polink-1 HRP DAB Detection systerm (Goat Anti-Rabbit IgG /HRP and 3\% $\mathrm{H}_{2} \mathrm{O}_{2}$ ), Lot No.K156911D, Normal goat serum working solution,WP140313 and Rabbit Anti-Goat IgG(H+L)/ HRP, Lot No.98028 were from ZhongShan Gold Bridge Biotechnology Co., Ltd. SDS-PAGE. Loading Buffer (reducing, 5×), CW0027A, CWBIO. Prolight HRP Chemiluminescent Kit, PA112, was from TIANGEN. Rabbit Anti-NPHS2/Podocin, Catalog No. BA1688, Lot No.13158, and DAB Color Development Kit, Lot No.AR1022, were from Boster. Pierce ${ }^{\circledR}$ Rapid ELISA mouse mAb Isotyping Kit, Lot No. PJ205664, was from Thermo Scientific.

\section{Animals and ethics statement}

Wistar rats were from Experimental Animal Center of Academy of Military medical Sciences SCXK (Jun)2012-0004, SD rats were from Vital River laboratory Animal Technology Co., Ltd. (SCXK-2012-0004). Animals experiments were carried out in the animal laboratory of Academy of Military Medical Sciences in compliance with the Institutional Animal Care and Use Program Guidelines. The animals were given food and water ad libitum, and housed under $12 \mathrm{hr} / 12 \mathrm{hr}$ light / dark cycle. All efforts were made to minimize suffering.

\section{Preparation of gold nanoclusters}

Gold nanoclusters (Au-NCs) were prepared by a previously reported method [30]. $\mathrm{HAuCl}_{4}$ in $\mathrm{BSA}$ solution was reduced by L-ascorbic acid and then $\mathrm{NaOH}$ was added to neutralize the residue acid. The reacted mixture was dialyzed in the PBS (Phosphate Buffered Saline) for further linkage with antibody.

\section{Preparation of BRCAA1 monoclonal antibodies}

BRCAA1 antigen, SSKKQKRSHK, has been 
identified as an antigen epitope located in BRCAA1 protein, which was highly expressed in the gastric cancer cells [31]. Using the recommended BRCAA1 protein as the immuno-antigen, the hybridoma cell line, S-200-5 had been cloned following the method of Afanassiev et al. [32]. BRCAA1 mAb, the monoclonal antibody excreted from S-200-5 cells was purified from mouse ascite by using protein G-Sepharose 4 fast flow (Amersham, Uppsala, Sweden). Its purity was more than $95 \%$. BRCAA1 mAb was identified to recognize BRCAA1 antigen with high specificity and affinity [33].

\section{BRCAA1 mAb conjugated with Au-NCs}

BRCAA1 conjugated Au-NCs were prepared by the carboxyl moieties of Au-NCs coupled to the amine groups of antibodies through $\mathrm{EDC} \cdot \mathrm{HCl}$ and NHS activated amide bond formation [33, 34]. Briefly, 10 mg Au-NCs was dissolved in $10 \mathrm{mM}$ MES buffer (pH $=5.4) 5 \mathrm{~mL} ; 1 \mathrm{mg}$ EDC and $1 \mathrm{mg}$ NHS were added, and the mixture was stirred gently at room temperature for $0.5 \mathrm{hr}$, then $2 \mathrm{mg}$ antibodies were added to the activated Au-NCs solution with continuous stirring in the dark at room temperature for $12 \mathrm{hr}$. At last, the mixture was treated with ultrafiltration (MWCO 3000 ) and concentrated, then stored at $4{ }^{\circ} \mathrm{C}$ for further experiments.

\section{Collection of blood and urine of rats treated with BRCAA1 mAb Au-NCs}

Wistar rats (180 g-220 g), 6 female and 6 male, were intravenously exposed to BRCAA1 mAb Au-NCs at the gold dose of $1.5 \mathrm{mg} / \mathrm{kg}$. Two rats were housed in one metabolism cage and they were given food and water ad libitum. The blood samples were collected at 13 time points including $0,2,10,30 \mathrm{~min}, 1,2,4,6,8$, 12, 24, 48 and $96 \mathrm{hr}$. At each time point after injected, blood was collected using a standard saphenous vein blood gathering technique. Urine samples were collected at 8 time points including before injection, $0-2,2-4,4-8,8-12,12-24,24-48$ and 48-96 hr.

\section{The determination of gold and antibody in the blood and urine samples}

The gold content in the blood and urine samples was measured with an inductively coupled plasma mass spectrometer (ICP-MS, type Agilent 7500 CE, Agilent Technologies, Waldbronn, Germany). Blood and urine samples were also diluted 5 times with distilled water for the TEM (HITACHI H-7650) observation.

Western blotting was employed to explore whether the BRCAA1 mAb conjugated with AuNCs could be excreted from kidney. Urine sample of BRCAA1mAb Au-NCs group was 6 times concentrated by hyperfiltration tube (Ultracel 30K, MILLIPORE Lot No: R3KA94103) by centrifugation (11000 rpm, $10 \mathrm{~min}$ ). $3 \mathrm{ml}$ urine was concentrated to $0.5 \mathrm{ml} .32 \mu \mathrm{l}$ concentrated urine sample was mixed with $8 \mu$ l SDSPAGE sample buffer. The mixture of $20 \mu \mathrm{l}$ BRCAA1 $\mathrm{mAb}(5 \mathrm{ng} / \mu \mathrm{l}), 12 \mu \mathrm{l}$ distilled water and $8 \mu \mathrm{l}$ SDSPAGE sample buffer was set as positive control. The above specimens were boiled for $5 \mathrm{~min}$ in order to make the protein denaturation completely. The proteins separated by $10 \%$ SDS-PAGE were transferred onto a nitrocellulose membrane. The membrane was blocked with $5 \%$ defatted milk powder in Tris-buffered saline containing $0.05 \%$ Tween 20 (TBST) and incubated one hour at $37{ }^{\circ} \mathrm{C}$ with horseradish peroxidase conjugated goat anti mouse IgG. The membrane was washed 5 times by TBST, reacted 3 min with chemoluminescence liquid away from light, and then scanned with Molecular Imager system (IMAGE QUAMT400, Bio RAD ChemiDoc $^{\mathrm{TM}} \mathrm{XRS}^{+}$).

In order to analyze the component of the antibody secreted, urine samples were modulated to $\mathrm{pH} 7.0$ with $0.5 \mathrm{M} \mathrm{NaH}_{2} \mathrm{PO}_{3}$ and then added with PBS to make the final 5 -fold dilution. The plasma samples were also 100 times diluted with PBS. The antibody isotypes in the diluted urine and plasma samples were analyzed by Pierce ${ }^{\circledR}$ Rapid ELISA mouse mAb Isotyping Kit following the kits instructions. Briefly, $50 \mu 1$ sample and $50 \mu \mathrm{l}$ Goat Anti-Mouse IgG + IgA + IgM - HRP Conjugate were mixed for 10 min and added to the well precoated with antibody specific for mouse IgG1, IgG2a, IgG3, IgA, IgM, kappa light chain and lambda light chain respectively. BRCAA1 $\mathrm{mAb}(250 \mathrm{ng} / \mathrm{ml})$ and BRCAA1 mAb Au-NCs $(10 \mu \mathrm{g} / \mathrm{ml})$ were set as positive controls. The plates were incubated for $1 \mathrm{hr}$ at room temperature and then washed 3 times with PBST (Phosphate Buffered Saline with Tween). $75 \mu 1$ of TMB substrate was added to each well, and 5 to 15 min later, $75 \mu$ of stop solution was added. The colored reaction product was measured at $450 \mathrm{~nm}$ on a microELISA Reader (Varioskan Flash version 2.4.3, Thermo Scientific, America).

\section{Influence on renal function and pathology in kidney}

Thirty female SD rats (180 g-220 g) were randomly divided into five groups (six in each group) containing control group, four experiment groups at 2, 6, 24 
and $48 \mathrm{hr}$ time points after BRCAA1 mAb Au-NCs treatment. Experimental groups were intravenously exposed to BRCAA1 mAb Au-NCs at the dose of $10 \mathrm{mg} / \mathrm{kg}$. Control group was treated with the same volume of saline. The urine samples were collected before experiment, 0-2, 2-6, 6-24 and 24-48 hr time frame. At each time point after injection, animals were anesthetized intraperitoneally with urethane at the dose of $1.2 \mathrm{~g} / \mathrm{kg}$. Their blood samples were collected by angio catheter exsanguination for blood serum biochemical detection. The kidney samples of rats were harvested. A half of one side kidney of each rat was fixed in $10 \%$ neutral buffered formalin, processed routinely to make paraffin section, and stained with hematoxylin and eosin (H\&E). The pathological change was recorded by a digital microscope (LEICA DM6000B). Some paraffin sections were used for immunohistochemisty analysis. Remanent kidney tissues of above were fixed with $2.5 \%$ glutaraldehyde in $0.03 \mathrm{M}$ potassium phosphate buffer $(\mathrm{pH}$ 7.4) for TEM analysis. The total protein in another kidney of each rat was extracted for Western blotting.

\section{Mechanism analysis of the renel excretion of BRCAA1 mAb Au-NCs}

Western blotting and immnohistochemistry were applied in order to analyze the possible mechanism of BRCAA1 mAb AuNCs. The total proteins of rat kidney were extracted by tissue protein extraction kit (Cat No: KGP2100) and quantified with DAB Color Development Kit. $50 \mu \mathrm{g}$ of Kidney total proteins in $20 \mu \mathrm{l}$ PBS buffer were mixed with $5 \mu \mathrm{l}$ SDS-PAGE sample buffer and boiled for $5 \mathrm{~min}$. Separated by $10 \%$ SDS-PAGE, the proteins were transferred onto a nitrocellulose membrane. The membrane was blocked with 5\% defatted milk powder in Tris-buffered saline containing $0.05 \%$ Tween 20 (TBST) and incubated with primary antibodies specific for ZO-1, Nephrin, Podocin and GAPDH at $4{ }^{\circ} \mathrm{C}$ overnight. The membrane was then incubated with horseradish peroxidase conjugated secondary antibodies and visualized with an enhanced chemiluminescence kit and a chemiluminescence imaging system. Densitometric analysis of band intensities was conducted using the image J 2X.

Immunohistochemistry was carried out using the LSAB 2 Kit (DakoJapan, Kyoto, Japan) by an antigen retrieval method. The primary antibodies were polyclonal rabbit anti rat ZO-1, Nephrin, Podocin. After deparaffinization, the sections were treated with methanol / hydrogen peroxide for 5 min to block endogenous peroxidase. In order to retrieve masked antigens, the slides were immersed in citrate buffer ( $\mathrm{pH}$ 6.0), and heated in an microwave oven for 5 $\min$ at $95^{\circ} \mathrm{C}$. The slides were incubated for $24 \mathrm{hr}$ at $4{ }^{\circ} \mathrm{C}$ with the primary antibody followed by a $30 \mathrm{~min}$ incubation with the biotinylated goat anti-mouse IgG, and then with peroxidase-labelled streptavidin for 30 min. Finally, 3, 3-diamino-benzidine was introduced to develop the colour, and haematoxylin was used for counterstaining. After dehydration, the sections were mounted with neutral resin.

\section{Results and Discussion}

\section{Au-NCs in blood and urine of rats}

After the BRCAA1 mAb Au-NCs were injected in vivo, the gold concentration in blood and urine was determined by ICP MASS. The result in Fig. 1 indicated that BRCAA1 mAb Au-NCs could quickly disperse in the blood. The high concentration of gold was measured in urine within $2 \mathrm{hr}$ after BRCAA1 mAb Au-NCs treatment. The gold concentrations in both blood and urine then decreased gradually to lower levels. At the time period of 48 to $96 \mathrm{hr}$, the gold concentration in blood sustained at the concentration of about $300 \mathrm{ng} / \mathrm{ml}$, whereas no gold in urine was detected at this period.

Aimed to know whether the gold determined in the blood and urine is from Au-NCs, the plasma and urine samples were directly observed by the transmission electron microscope (TEM). The TEM images of the NCs in blood and urine gave microscopic insight for the distribution of gold nanoparticles. The nanoparticals in blood and urine clearly indicated that higher concentration of gold clusters appeared in the plasma sample of $2 \mathrm{hr}$ time point and particles of the same size were found in the urine samples during 2 to $48 \mathrm{hr}$ time points (Fig. 2, 3). This result elucidated that gold clusters could be excreted through glomerular filtration in good agreement with the above results (Fig. 1).

From the detection above we concluded that after BRCAA1 Au-NCs were injected, the gold particles were quickly excreted from kidneys. However, we still could not conclude if the excreted gold clusters or some of them were still ligated with BRCAA1 mAb. At first, we used Western blotting to detect the antibody in urine. The proteins in urine samples were respectively separated by non-reduced SDS PAGE and reduced 

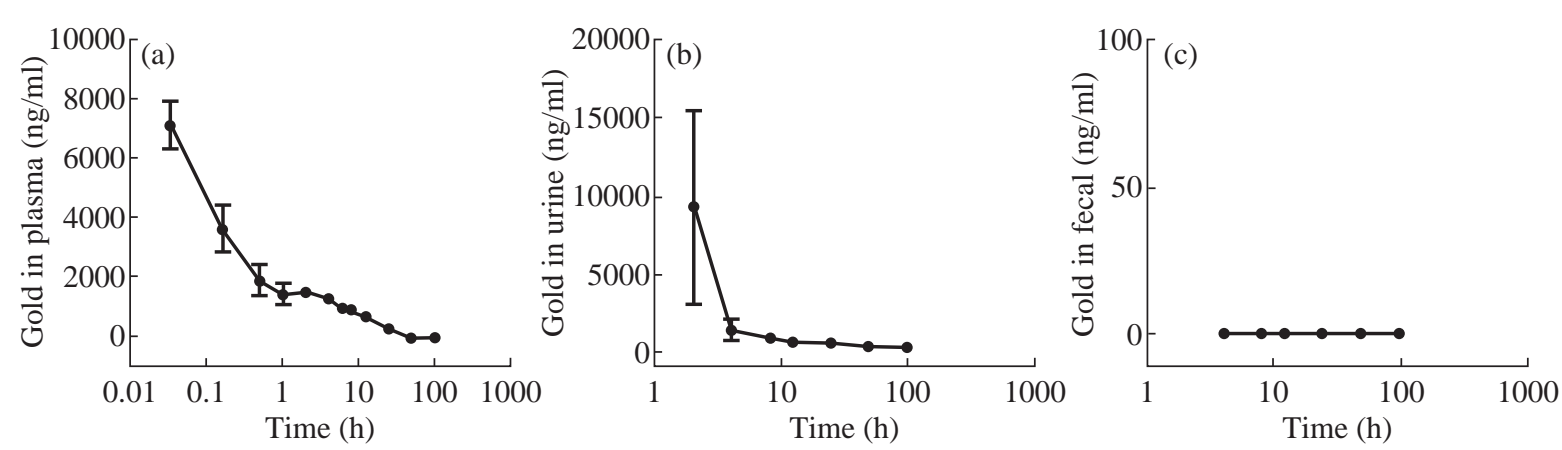

Fig. 1 Gold concentrations in the plasma, urine and fecal of rats administrated with BRCAA1 mAb Au-NCs. Wistar rats, 6 male and 6 female, were in vivo injected with BRCAA1 mAb Au-NCs at a dose of $1.5 \mathrm{mg} / \mathrm{kg}$. At different time points, the plasma, urine and fecal samples were collected and their gold concentrations were measured with an inductively coupled plasma mass spectrometer. Data are presented as means \pm standard deviations, $\mathrm{n}=12$.

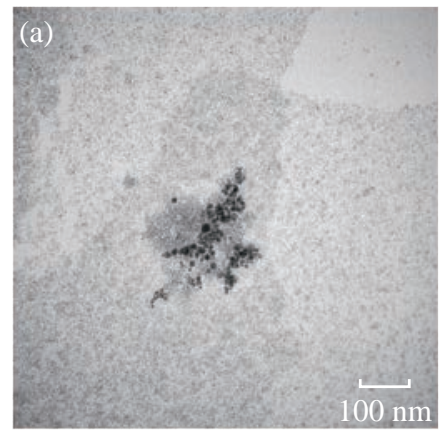

Bar: $100 \mathrm{~nm}$

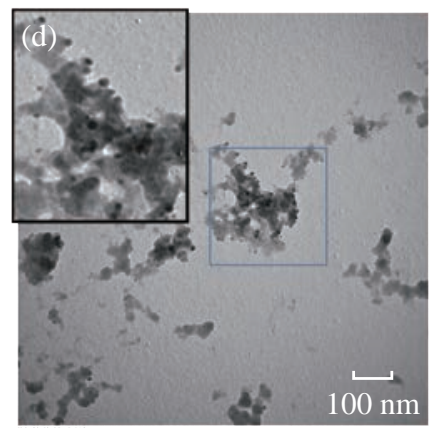

Bar: $100 \mathrm{~nm}$

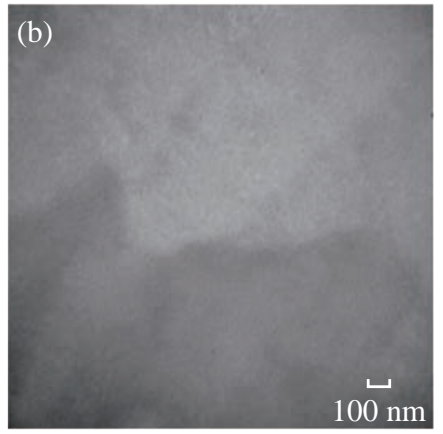

Bar: $100 \mathrm{~nm}$

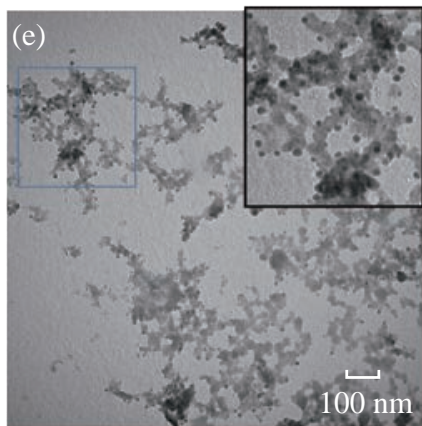

Bar: $100 \mathrm{~nm}$

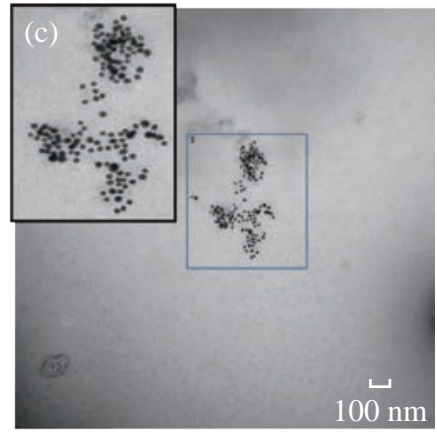

Bar: $100 \mathrm{~nm}$

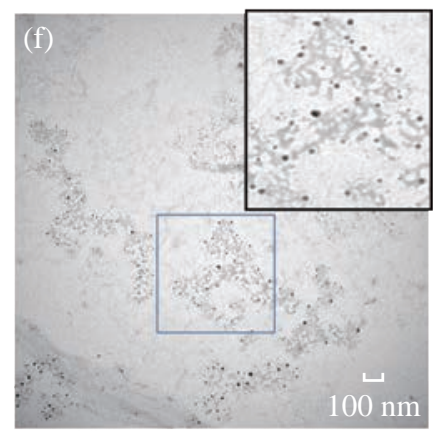

Bar: $100 \mathrm{~nm}$

Fig. 2 Glod nanoparticles in the plasma of rats treated with BRCAA1 mAb Au-NCs. Wistar rats, 6 male and 6 female, were in vivo injected with BRCAA1 mAb Au-NCs at a dose of $1.5 \mathrm{mg} / \mathrm{kg}$. At different time points the plasma samples were collected and mixed. The plasma samples were diluted 5 times with saline for TEM observation. (a) BRCAA1 mAb Au-NCs in saline; (b)-(f) Plasma collected at $0,2,6,24,48 \mathrm{hr}$ time points. Scale bars $=100 \mathrm{~nm}$ in each part.

SDS PAGE. Then the translated antibody on membrane was recognized by HRP conjugated goat against mouse IgG. The results of Western blotting indicated that in the urine samples of rats having received BRCAA1 mAb Au-NCs, the whole antibody (Fig. 4(a)) and the Fc fragment (Fig.4(c)) were recognized by HRP-GAMIgG. We randomly selected four samples at 4-8 $\mathrm{hr}$ and $8-12 \mathrm{hr}$ to detect whether the positive band was from the individual animal, and the result showed that the antibody presented in most urine samples of BRCAA1 $\mathrm{mAb} \mathrm{Au}-\mathrm{NCs}$ treated rats (Fig. 4(b) and (d)).
From the Western blot result above we observed the antibody excretion from kidneys. We still could not confirm whether the secreted antibodies included BRCAA1 mAb. We analyzed BRCAA1 mAb using mouse antibody isotype ELISA kit and found that its isotype was $\operatorname{IgG} 2 b$. So we analyzed the antibody constitution in the plasma and urine samples. Firstly, we determined the background mouse antibody signals in the plasma and urine of control rats. The result showed that in the control samples, no mouse antibody signals in urine and lower mouse antibody signals of 


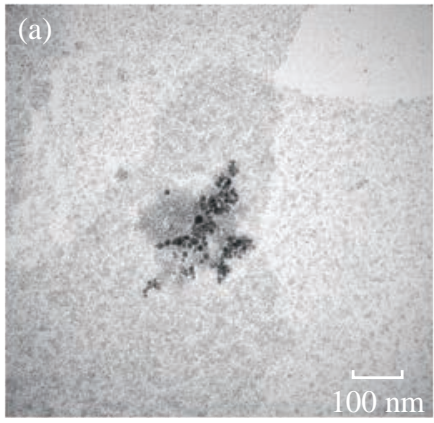

Bar: $100 \mathrm{~nm}$

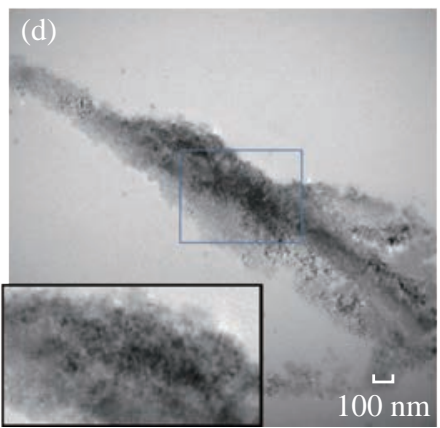

Bar: $100 \mathrm{~nm}$

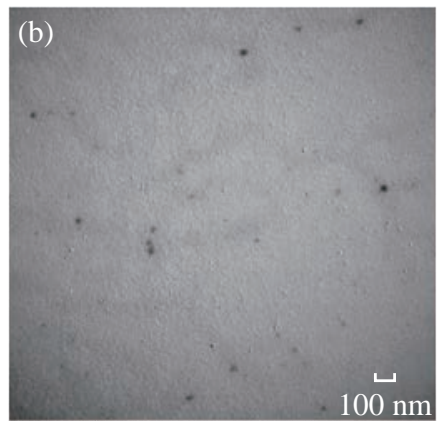

Bar: $100 \mathrm{~nm}$

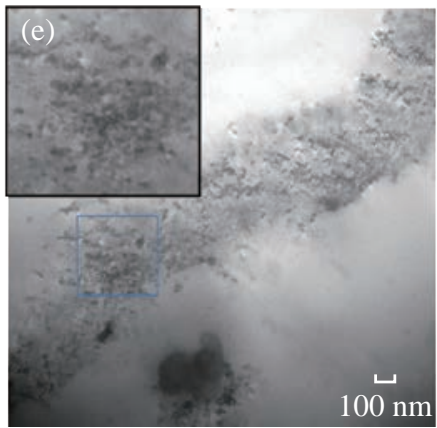

Bar: $100 \mathrm{~nm}$

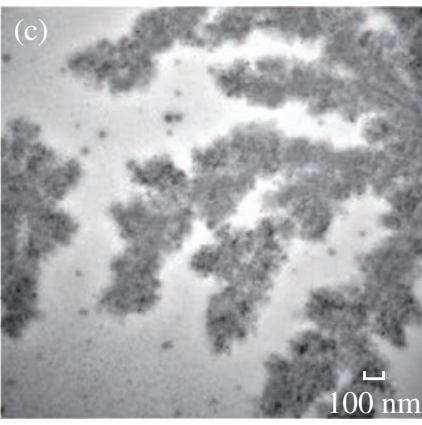

Bar: $100 \mathrm{~nm}$

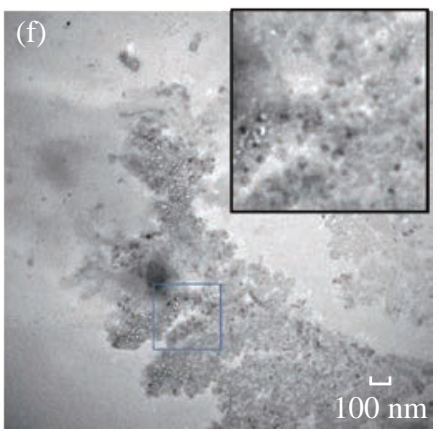

Bar: $100 \mathrm{~nm}$

Fig. 3 Gold nanoparticles in the urine of rats treated with BRCAA1 mAb Au-NCs. Wistar rats, 6 male and 6 female, were in vivo injected with BRCAA1 mAb Au-NCs at a dose of $1.5 \mathrm{mg} / \mathrm{kg}$. At different time points the urine samples were collected and mixed. The urine samples were concentrated 5 times by super-filtration for TEM observation. (a) BRCAA1 mAb Au-NCs in saline; (b)-(f) Urine collected at 0, 0-2, 2-6, 6-24, 24-48 hr time points. Scale bars $=100 \mathrm{~nm}$ in each part.

(a)
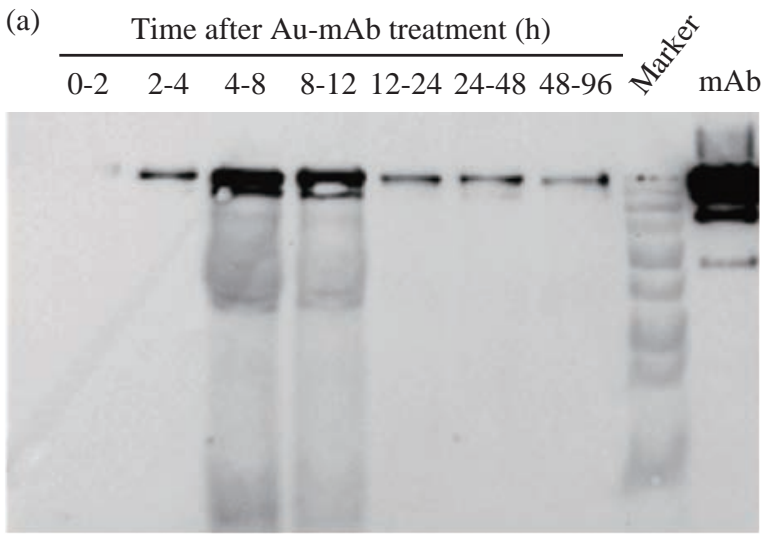

(c)

Time after Au-mAb treatment (h)

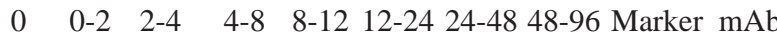

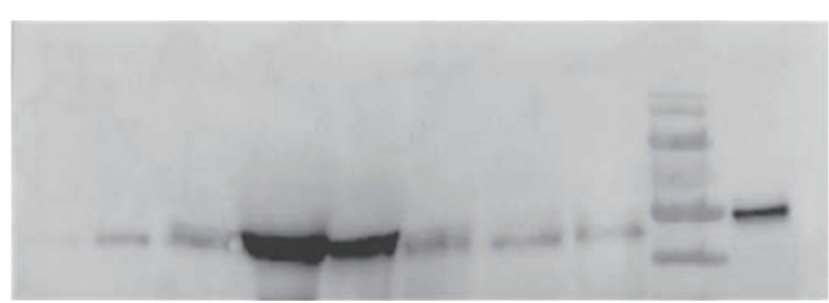

(b)

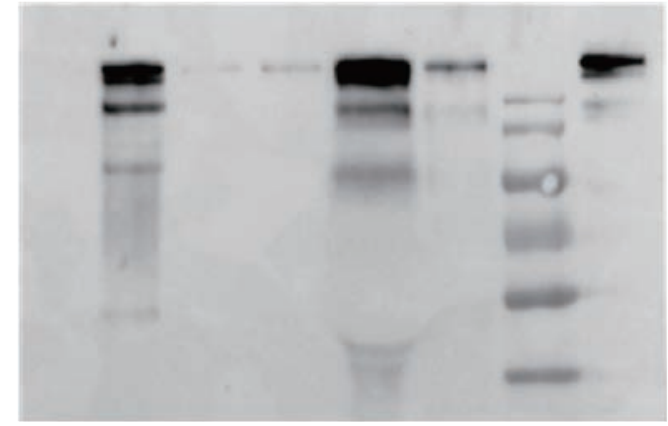

(d)

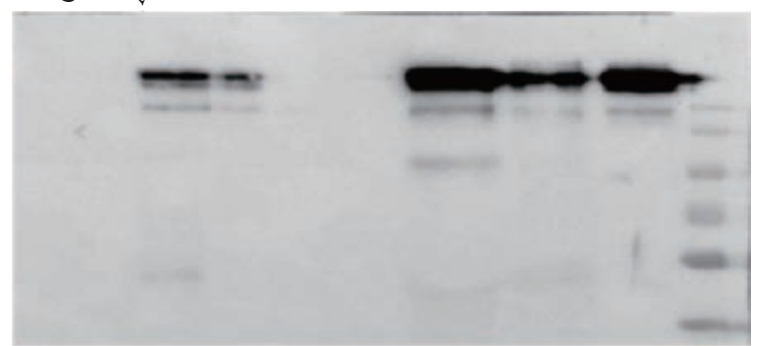

Fig. 4 Western Blotting determination of antibody in urine samples of rats administrated with BRCAA1 mAb Au-NCs. Urine samples were concentrated by super-filtration and separated by $12 \%$ non-reduced ((a), (b) and (d)) and reduced SDS PAGE (c). The proteins translated onto nitrocellulose membrane were recognized by HRP-GAM-IgG. BRCAA1 (Ab) and BRCAA1 mAb Au-NCs (Au-Ab) were set as positive control. Equal volume of urine sample at the same time point were mixed and to be analyzed ((a) and (c)). At 4-8 $\mathrm{hr}$ and $8-12 \mathrm{hr}$ time points, four urine samples were selected randomly to be determined. 
IgG3, IgM and kappa chain in plasma were observed. When rats were treated with BRCAA1 mAb Au-NCs, the concentration of gold was about $2000 \mathrm{ng} / \mathrm{ml}$ in their plasma of $2 \mathrm{hr}$ time point in which the mouse antibody signals were difficult to be monitored. This result was predictable because BRCAA1 mAb Au-NCs, even at $10,000 \mathrm{ng} / \mathrm{ml}$ in saline, could not give strong antibody isotype signals due to the linkage of antibody with gold nanoparticals (Fig. 5(b)).

The mouse antibody's isotype in urine of rats gave
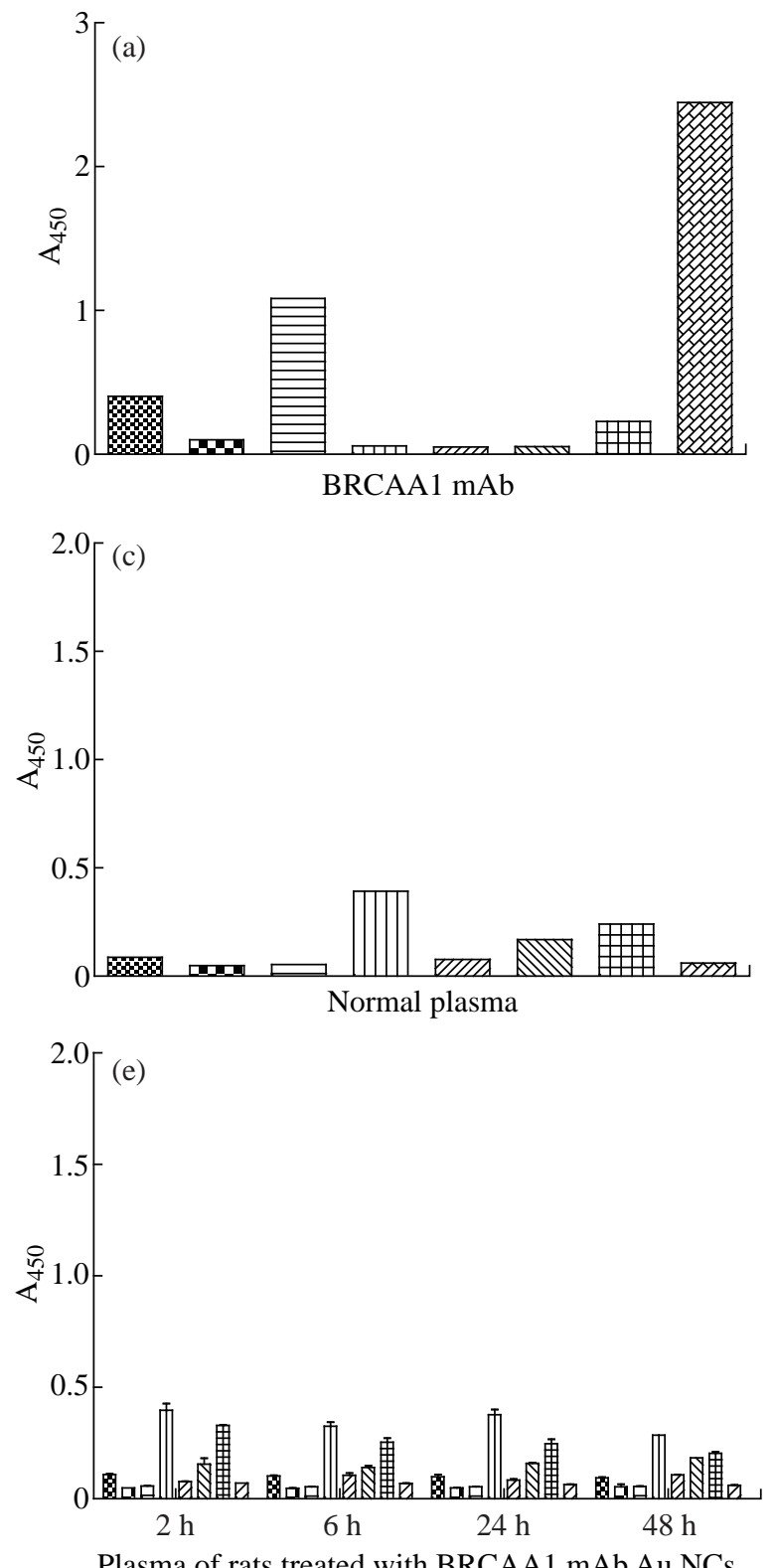

Plasma of rats treated with BRCAA1 mAb Au NCs

\& IgG1, $\mathrm{R}$ IgG2a, us very interesting information. In the period of 0 to $6 \mathrm{hr}$ after BRCAA1 mAb Au-NCs administration, high concentration of gold nanoparticles secreted in urine samples (Fig. 1(b)). As the result from plasma of administrated rats, $\operatorname{IgG} 2 \mathrm{~b}$, the isotype of BRCAA1, was difficult to be monitored in the urine samples. However, strong signals of lambda chain and kappa chain were observed in 2-6 hr urine samples. The lambda chain should be from BRCAA1 mAb, because kappa chain was the main light chain of rat antibodies
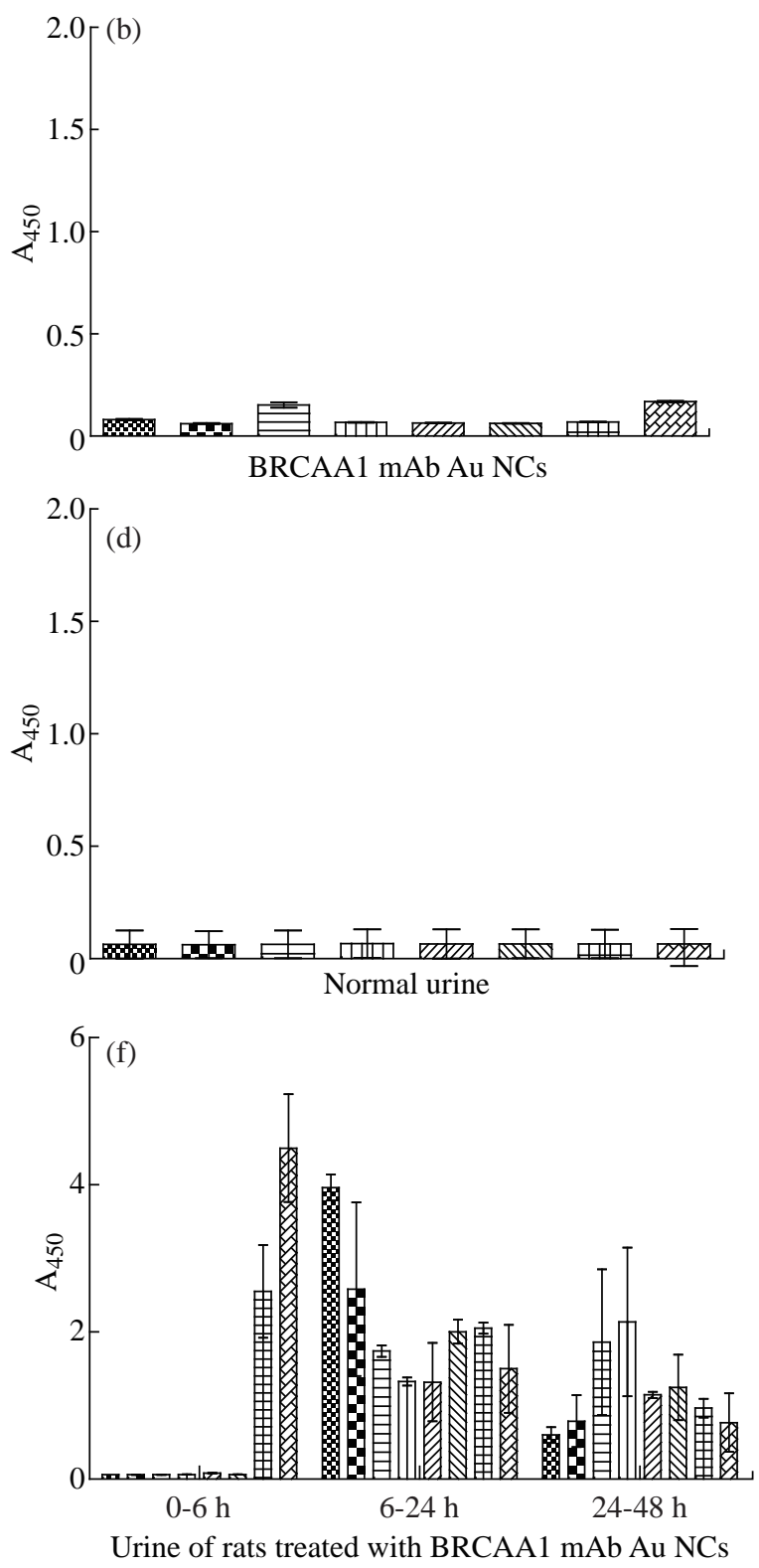

Urine of rats treated with BRCAA1 mAb Au NCs

Fig. 5 Antibody isotype in urine samples of rats administrated with BRCAA1 mAb Au-NCs. ELISA was used to analyze the isotype of BRCAA1 mAb in saline. Mouse IgG1, IgG2a, IgG3, IgA, IgM, kappa light chain and lambda light were captured by coating specific antibody and recognized by Goat Anti-Mouse IgG + IgA + IgM - HRP Conjugate. (a) BRCAA1 mAb in saline; (b) BRCAA1 mAb Au-NCs in saline; (c) antibody in normal plasma of rats; (d) antibody in normal urine of rats; (e) antibody in plasma of rats treated with BRCAA1 mAb Au-NCs; (f) antibody in urine of rats treated with BRCAA1 mAb Au-NCs. Data are presented as means \pm standard deviations, $\mathrm{n}=4$. 
in plasma. Comparing with the lower background of antibody signals in the normal urine, this result demonstrated that the filtration of kidneys was affected at this time and the affection was even more serious during 6 to $48 \mathrm{hr}$ period, because besides lambda and kappa chains, IgG1, IgG2a, IgG2b, IgG3, IgA and IgM were detected. So we concluded that BRCAA1 mAb was not the only source of the strong antibody signals, and we wanted to know what injures had happened in the glomcrulus of rats inducing the leakage of big molecules passing through the filtrating blockage.

\section{Urinalysis and biochemistry}

Urinalysis and biochemical analyses were programed to reveal the potential toxicity of the BRCAA1 mAb Au-NCs. The protein concentrations in urine were determined and the proteinuria was found in rats injected with BRCAA1 mAb NCs for 6-24 and 24-48 $\mathrm{hr}$ (data not shown). We also presented the biochemistry results in the plasma of rats including alanine transaminase (ALT), aspartate transaminase (AST), albumin (ALB), blood urea nitrogen (BUN), creatinine (CREA), total bilirubin (TBIL), creatinine (CREA), creatinekinase (CK), albumin (ALB), alkaline phosphatase (ALP), total protein (TP), cholesterol (CHOL), blood glucose (GLU) and triglyceride (TG) (Table 1). We paid more attention on ALT, AST, BUN, TBIL, CK and CREA which are related to the functions of liver and kidney closely.
Increasing in the levels of ALT, AST, TBIL and BUN, and decreaseing in the level of CK were found at $2 \mathrm{hr}$ after the treatment. Distributed in liver cells, the ALT mainly exists in the cytoplasm, and the AST mainly exists in the cytoplasm and mitochondria. The increase of their activities frequently reminds the potential hepatoxicity [35]. TBIL concentration in blood is related to the excreting ablility of bilirubin from liver to blood [36]. The increasing level of BUN usually indicates the nephrotoxicity. These biochemical index changes within $48 \mathrm{hr}$ after BRCAA1 mAb Au-NCsAuNCs treatment showed that these nanoparticals might induce the reversible hepatoxicity and nephrotoxicity. The mechanism of the decrease in CK levels requires further investigation.

\section{Histopathological analysis}

The Electron microscope image of kidney in Fig. 6 shows some structural injury of the kidney. Through the ultrastructural organization, the capillaries endotheliocyte damage was found in kidney glomerulus. The damage lesion was directly related to the exposure time. The pathological analysis also showed injuries in the kidney glomerulus. As shown in Fig. 7, after $2 \mathrm{hr}$ of BRCAA1 mAb Au-NCs injected, apparent pycnosis, karyorrhexis and hyalomitome acidophilia enhancement were found in some vascular endothelial cell nuclei. Necrosis of part of the vascular endothelial cell and enhancement of the mesangial cell

Table 1 Effect of BRCAA1 mAb Au-NCs on nephrotoxicity and hepatotoxicity related biochemical indicators in sera of mice

\begin{tabular}{|c|c|c|c|c|c|}
\hline \multirow{2}{*}{ Parameters } & \multicolumn{5}{|c|}{ Time after BRCAA1 mAb Au-NCs treatment (h) } \\
\hline & 0 & 2 & 6 & 24 & 48 \\
\hline $\operatorname{ALT}(\mathrm{U} / \mathrm{L})$ & $44.0 \pm 3.6$ & $112.0 \pm 21.9^{* *}$ & $151.0 \pm 56.6^{* * *}$ & $55.7 \pm 14.5$ & $32.2 \pm 3.4$ \\
\hline $\operatorname{AST}(\mathrm{U} / \mathrm{L})$ & $135.3 \pm 26.1$ & $335.0 \pm 80.4^{* *}$ & $311.3 \pm 95.3^{* *}$ & $216.5 \pm 87.5$ & $125.3 \pm 14.3$ \\
\hline $\operatorname{ALP}(\mathrm{U} / \mathrm{L})$ & $200.3 \pm 43.0$ & $224.2 \pm 31.4$ & $221.6 \pm 15.4$ & $193.2 \pm 50.8$ & $213.2 \pm 24.6$ \\
\hline $\mathrm{TP}(\mathrm{g} / \mathrm{L})$ & $69.1 \pm 3.0$ & $65.2 \pm 5.8$ & $70.6 \pm 4.0$ & $59.3 \pm 2.1^{* *}$ & $61.2 \pm 4.0^{*}$ \\
\hline $\operatorname{ALB}(g / L)$ & $35.9 \pm 1.4$ & $35.1 \pm 3.6$ & $36.4 \pm 3.5$ & $28.1 \pm 0.8^{* * *}$ & $28.2 \pm 2.4^{* *}$ \\
\hline TCHO (mM) & $2.1 \pm 0.2$ & $1.7 \pm 0.0^{*}$ & $2.5 \pm 0.4$ & $2.2 \pm 0.3$ & $2.2 \pm 0.2$ \\
\hline BUN (mM) & $7.0 \pm 0.5$ & $8.9 \pm 0.6^{*}$ & $9.7 \pm 0.5^{* *}$ & $9.8 \pm 1.6^{* *}$ & $9.7 \pm 1.3^{* *}$ \\
\hline TBIL (mM) & $0.0 \pm 0.0$ & $0.7 \pm 0.2$ & $2.8 \pm 1.3^{* * *}$ & $2.1 \pm 0.7^{* * *}$ & $0.4 \pm 0.1$ \\
\hline GLU(mM) & $9.9 \pm 0.7$ & $7.6 \pm 0.6^{*}$ & $6.2 \pm 1.3^{* * *}$ & $7.3 \pm 0.8^{* *}$ & $11.9 \pm 1.7^{*}$ \\
\hline TG (mM) & $0.8 \pm 0.2$ & $1.8 \pm 0.3^{* * *}$ & $0.9 \pm 0.2$ & $0.7 \pm 0.1$ & $0.7 \pm 0.2$ \\
\hline CREA (mM) & $21.8 \pm 1.4$ & $26.0 \pm 2.8^{* *}$ & $23.5 \pm 1.2$ & $23.9 \pm 1.5$ & $25.1 \pm 1.6^{*}$ \\
\hline CK (U/L) & $4442.3 \pm 432.5$ & $1044.2 \pm 253.6^{* * *}$ & $741.7 \pm 425.3^{* * *}$ & $4449.8 \pm 995.5$ & $4793.5 \pm 427.0$ \\
\hline
\end{tabular}

Alanine aminotransferase (ALT), aspartate aminotranferase (AST), alkaline phosphatase (ALP), total protein (TP), albumin (ALB), total cholesterol (TCHO), blood urea nitrogen (BUN), total bilirubin (TBIL), glucose (GLU), triglycerides (TG), creatinine (CREA) and creatinekinase (CK) in rat sera were determined. Data were expressed as mean \pm S.D. $\mathrm{n}=5 ;{ }^{*} P<0.05$, compared with normal control; ${ }^{* *} P<0.01,{ }^{* * *} P<0.001$, compared with normal control. Significant difference was calculated by SPSS18.0 


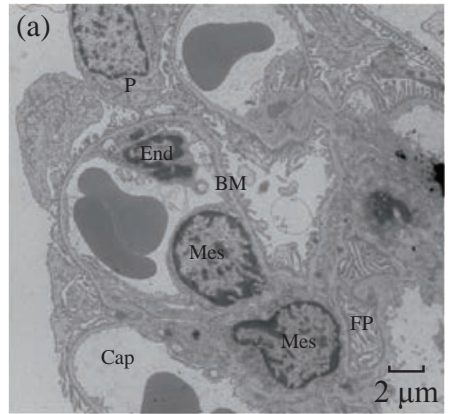

Bar: $2 \mu \mathrm{m}$

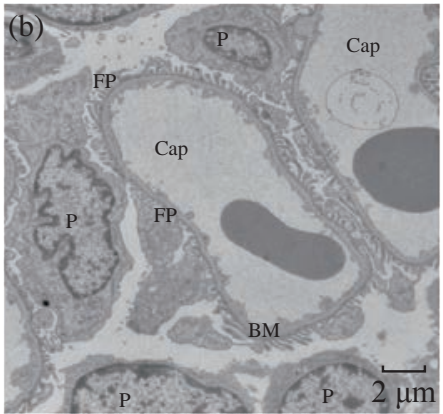

Bar: $2 \mu \mathrm{m}$

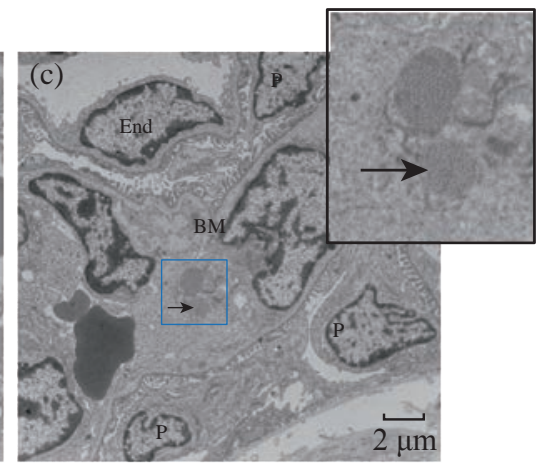

Bar: $2 \mu \mathrm{m}$

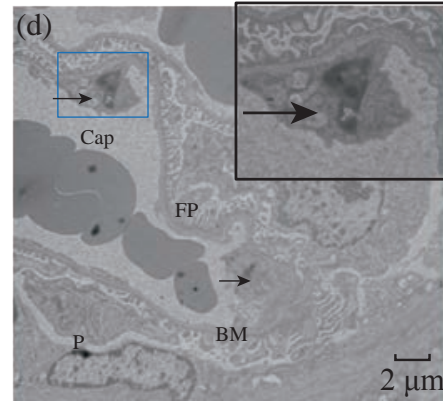

Bar: $2 \mu \mathrm{m}$

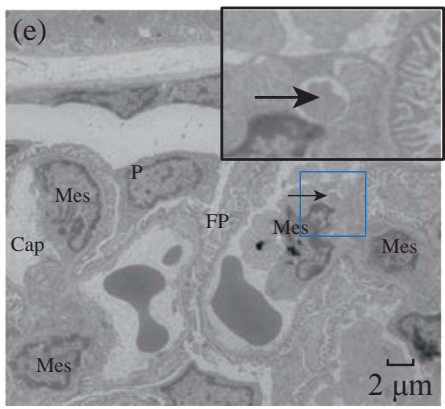

Bar: $2 \mu \mathrm{m}$

Fig.6 Electron microscope image of kidneys of rats treated with BRCAA1 mAb Au-NCs. The microscope image of kidney was observed by TEM. Some capillaries endothelicyte damage in glomerulus was found after BRCAA1 mAb Au-NCs treatment. (a)(e) Time points 0, 2, 6, 24, $48 \mathrm{hr}$ after the administration. Cap, capillary lumen; End, vascular endothelial cell; P, podocyte; FP, foot process; BM, basal membrane; Mes, intercapillary cells. In all sections detected, no significant injuries in the basal membrane and foot process were found. The main injure induced by BRCAA1 mAb NCs was the damage of endotheliocyte neorobiosis indicated by arrows. (c) Individual endotheliocyte nerobiosis in the blood vessel loop, lumens collapse, ground substance increasing. (d) Endotheliocyte neorobiosis induced the exposing of basal membrane and the fusing of adjacent capillary lumens. (e) Endotheliocyte neorobiosis, broad of capillary lumen, the fragments of neorobiosised cells were phagocytosed by intercapillary cells, more of base materials under basal membrane.
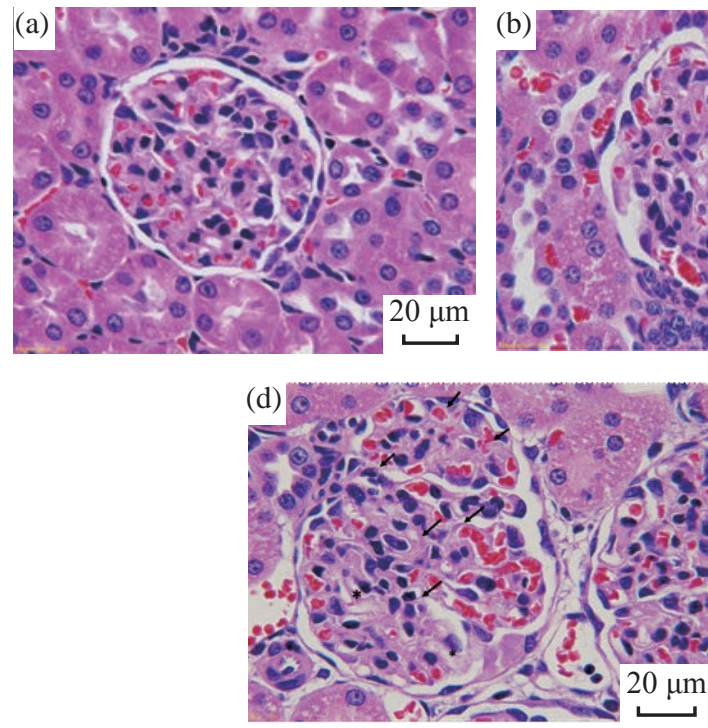
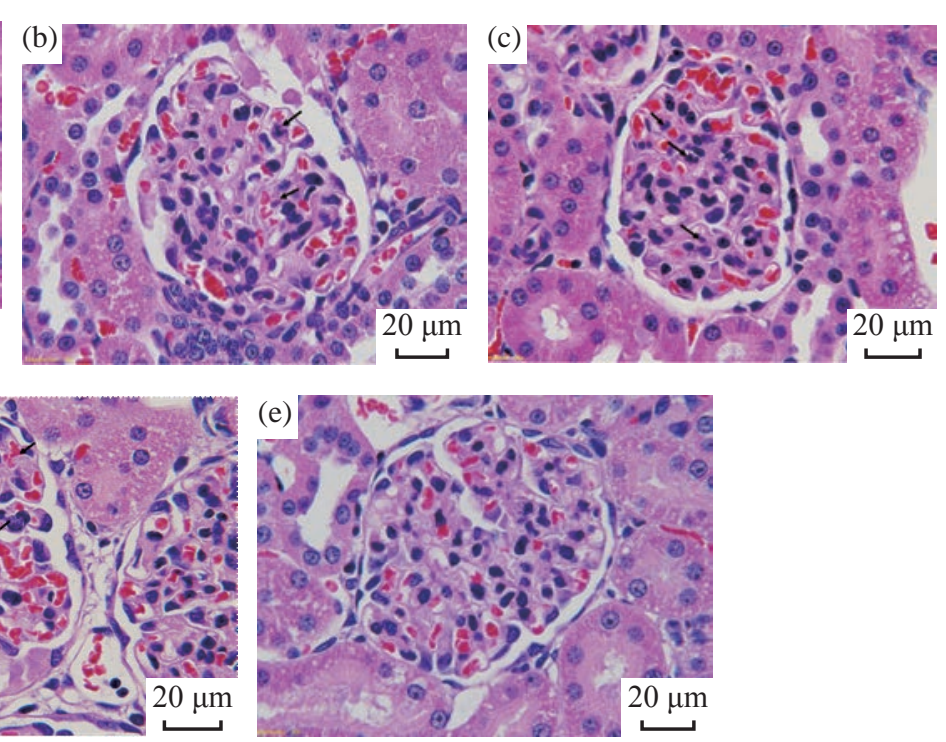

Fig. 7 Pathological changes in kidney glomerulus of rats treated with BRCAA1 mAb Au-NCs. Microscopic observation was performed on sections of tissue samples under a Leica DMI6000 B fluorescence microscope, and photographs were taken using Leica Application Suite V3. Scale bar $=20 \mu \mathrm{m}$ in each part. (a)-(e) Time points 0, 2, 6, 24, 48 hr. Pathological changes were observed in glumerulus. (b) Arrows indicated the karyopyknosis, karyorrhexis and increased karyorrhexis were found in individual endotheliocyte. (c) Some endotheliocyte necrosis and more intercapilary cells aggregated to the necrosis cells to deserve their phagocytose function. (d) More endotheliocyte necrosis cells were found in the glomerulus. Intercapilary cells deserved their phagocytose activity. Basal material increased in mesangial region. Some vascular net was not integrated (labeled by *). Original magnification $1000 \times$. 
phagocytize function had been seen at $6 \mathrm{hr}$ to $48 \mathrm{hr}$ after injection. These results indicate that the kidney suffered from the microscopic damage.

\section{Western blotting and immunohistochemisty}

Podocytes are specialized epithelial cells of the kidney filtration barrier that contribute to permselectivity via a series of interdigitating actinrich foot processes. A unique cell junction positioned among adjacent projections is slit diaphragm [37]. Nephrin and Podocin are two important proteins of podocytes to maintain normal slit diaphragm structure. The critical role of nephrin has been confirmed by different animal models with nephrin knockout and knockdown [38]. Nephrin is connected to the actin cytoskeleton by several adapter proteins and makes a pivotal contribution to the regulation of podocyte actin dynamics [39]. As well known, podocin presents the

(a)
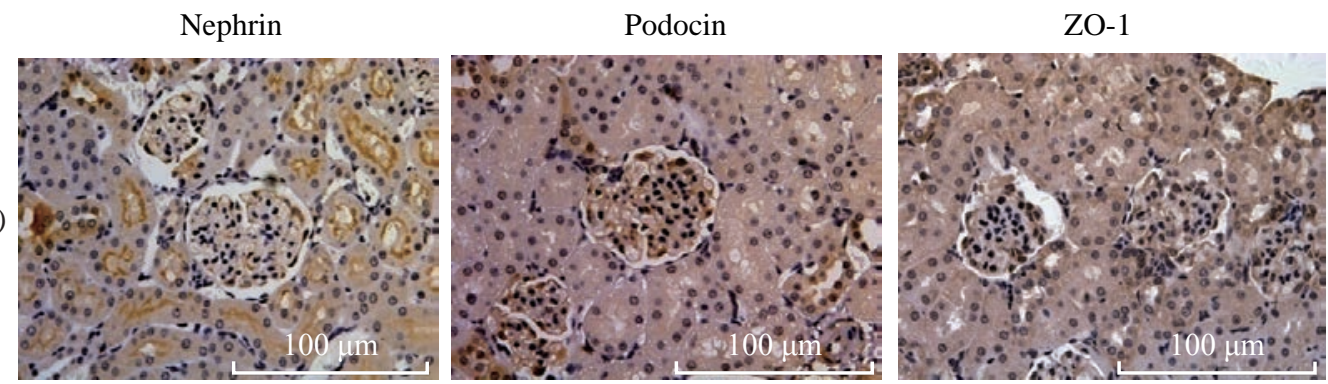

(b)
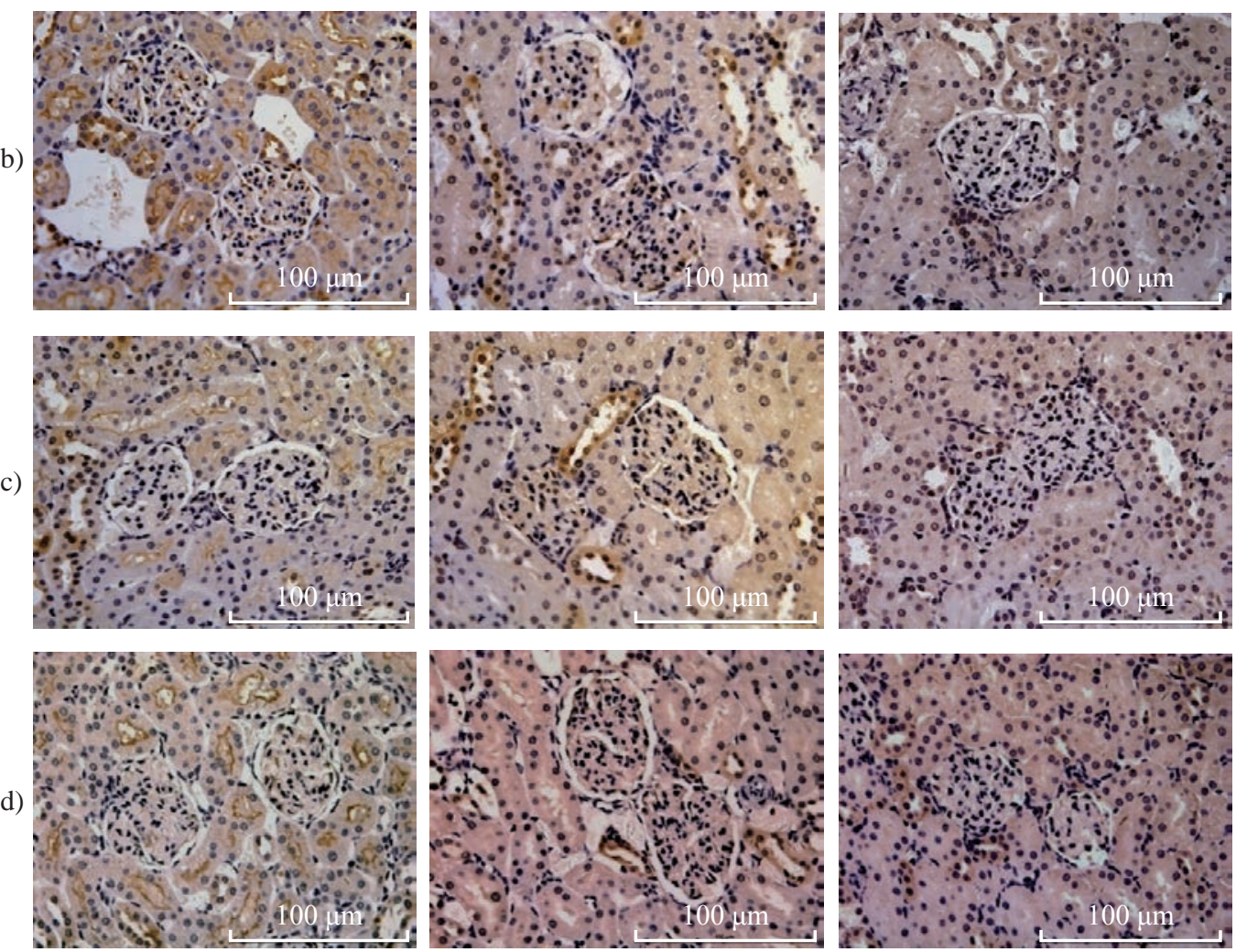

(e)
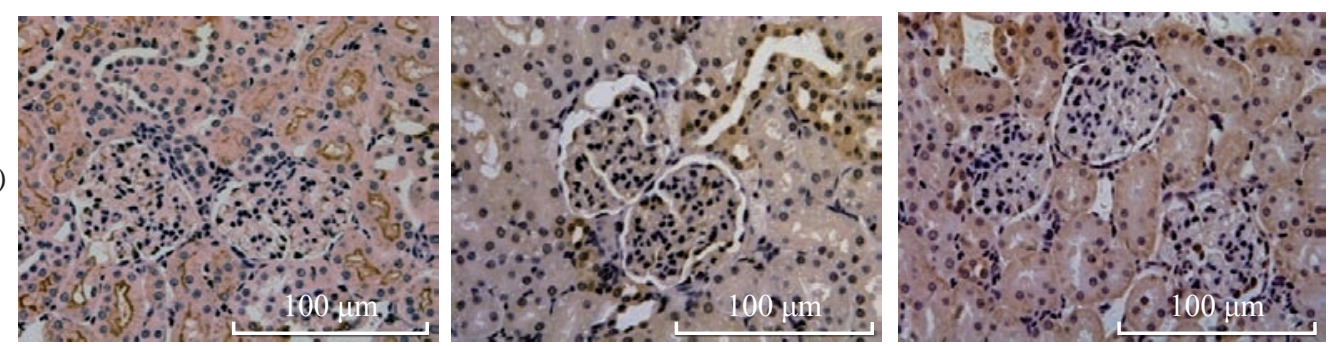

Fig. 8 Immunohistochemical staining of nephrin, podocin and ZO-1 in kidney glomerulus of rats treated with BRCAA1 mAb AuNCs. Nephrin, podocin and ZO-1 in kidney were respectively recognized by specific antibody following reacted with the biotinylated goat anti-mouse IgG. Scale bar $=100 \mu \mathrm{m}$ in each part. (a) From normal control rat; (b)-(e) From rats administrated with BRCAA1 $\mathrm{mAb}$ Au-NCs at 2, 6, 24 and $48 \mathrm{hr}$ after the nano-particles exposure. Original magnification $400 \times$. 
similar function to nephrin. Reduction of nephrin and podocin expression is often identified in adult kidney diseases. ZO-1 is a key protein that is specific for the tight junction or zonula occludens. The cells formed homogeneous monolayers with well-expressed ZO-1 protein [40]. The tight junctions could not be well formed among sells lacking ZO-1, for instance, MDCK cells showed higher sensitivity to the barrier loss induced by actin depolymerization [41].

In order to further reveal the close relationship between the proteinuria and the integrality of tight junction and slit membrane, we assessed the expression levels of these proteins by immunohistochemistry using specific antibody that recognizing ZO-1, Nephrin and Podocin respectively. As shown in Fig. 8, we observed that all of the expressing levels of ZO-1, Nephrin and Podocin decreased from $6 \mathrm{hr}$ after the exposure of BRCAA1 mAb Au-NCsAu-NCs. It was in accordance with the result of Western blot shown in Fig. 9. Both of the results from the immunohistological evaluation and the Western blot determination showed that the change in fitration fuction of renal glomerulus might be related to the depressed protein levels of Zo-1, Nephrin and Podocin after the treatment of BRCAA1 mAb Au-NCs. The Gold nanomaterials have long been considered as possible drug delivery vehicles applicable to therapy of a range of diseases. They can be easily synthesized in a variety of shapes with size ranging from $1 \mathrm{~nm}$ to more than $100 \mathrm{~nm}$ [43-46]. Developing functional nanomaterials with efficient renal clearance is of fundamental importance to their in-vivo biomedical applications [46-49]. Ideal nanomaterial should be effectively cleared out of the body, have little accumulation in organs. Sub-5.5 $\mathrm{nm}$ particles can pass through the barrier for renal clearance [50]. However, we need to know whether these particles still could pass through glomerulus when they are functioned by connecting with other molecules such as antibodies. BRCAA1 mAb conjugated Au-NCs were the gold clusters with the diameter of $5 \mathrm{~nm}$ functioned with BRCAA1mAb, an antibody that could recognize stomach cancer cells. These particles were much bigger than the original ones. In the urine of rats injected with Au BRCAA1 mAb NCs in vivo, we found gold nanoparticles that could be measured by ICP Mass and observed by TEM. The result of Western blot and ELISA indicated that BRCAA1 mAb was still linked with gold clusters by renal clearing even at $2 \mathrm{hr}$ after the administration. In the urine samples within $6 \mathrm{hr}$, free light chains, $\lambda$ from BRCAA 1 and $\kappa$ from rat blood, were determined. This result indicated that functioned gold clusters could pass through glomerulus quickly and affect the filtration barrier inducing the leakage of light chains firstly and followed by the excretion of big antibodies. These excreated molecules contained not only the disjuncted BRCAA1 mAb and

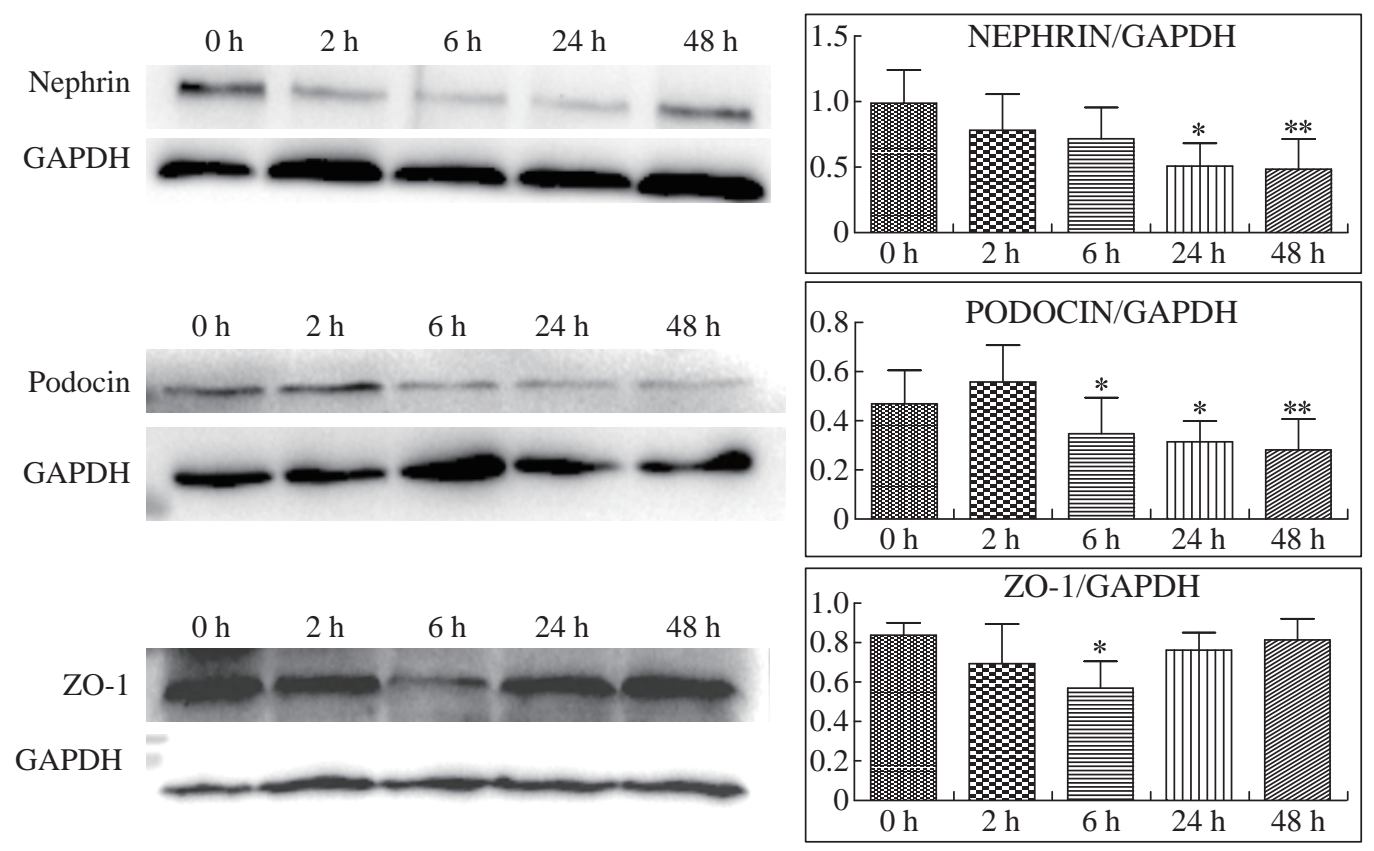

Fig. 9 Western blotting analysis of nephrin, podocin and ZO-1 proteins in kidneys of rats treated with BRCAA1 mAb Au-NCs. Nephrin, podocin and ZO-1 proteins of renal tissue form 0, 2, 6, 24 and $48 \mathrm{hr}$ after BRCAA1 mAb Au-NCs exposures were analyzed by Western blotting. Values are the mean $\pm \mathrm{SD}, \mathrm{n}=3$. Nephrin: ${ }^{*} \mathrm{P}<0.05$ versus $0 \mathrm{hr},{ }^{* *} \mathrm{P}<0.01$ versus $0 \mathrm{hr}$; Podocin: ${ }^{*} P<0.05$ versus $2 \mathrm{hr},{ }^{* *} \mathrm{P}<0.01$ versus $2 \mathrm{hr} ; \mathrm{ZO}-1:^{*} \mathrm{P}<0.05$ versus $0 \mathrm{hr}$. 
its degraded fragments, but also the molecules in the rat blood. Using HRP labeled goat againt rat IgG, we determined the presence of rat antibody in the 6 hr urine sample of treated rats by Western blot (data not shown). The excretion of big molecules from kidneys is related to the influence of gold clusters on the glomerulus structure. We have observed the down regulated expression of three proteins, ZO-1, Nephrin and Podocin. As for why their levels were depressed and whether there were other mechanism related to this secretion need further studies in future.

\section{Acknowledgements}

This work was supported by National Natural Scientific Fund (No.81327002), the National Key Basic Research Program (973 Project) (No. 2015CB931802 and No. 2010CB933901), 863 Project of China (2014AA020700), and Shanghai Science and Technology Fund (No.13NM1401500).

\section{References}

[1] J. Xie, Y. Zheng, and J.Y. Ying, Protein-directed synthesis of highly fluorescent gold nanoclusters. $J$ Am Chem Soc, 2009, 131(3): 888-889.

[2] H. Wei, Z. Wang, J. Zhang, et al., Time-dependent, protein-directed growth of gold nanoparticles within a single crystal of lysozyme. Nat Nano, 2011, 6(2): 93-97.

[3] S. Mayavan, N.K. Dutta, N.R. Choudhury, et al., Selforganization, interfacial interaction and photophysical properties of gold nanoparticle complexes derived from resilin-mimetic fluorescent protein rec1-resilin. Biomaterials, 2011, 32(11): 2786-2796.

[4] X. Le Guevel, N. Daum, M. Schneider, Synthesis and characterization of human transferrin-stabilized gold nanoclusters. Nanotechnology, 2011, 22(27): 275103.

[5] Y. Shichibu, Y. Negishi, H. Tsunoyama, et al., Extremely high stability of glutathionate-protected Au25 clusters against core etching. Small, 2007, 3(5): 835-839.

[6] A.R. Garcia, I. Rahn, S. Johnson, et al., Human insulin fibril-assisted synthesis of fluorescent gold nanoclusters in alkaline media under physiological temperature. Colloids Surf B Biointerfaces, 2013, 105: 167-172.

[7] F. Wen, Y. Dong, L. Feng, et al., Horseradish peroxidase functionalized luorescent gold nanoclusters for hydrogen peroxide sensing. Anal Chem., 2011, 83: 1193-1196.

[8] M. Balamurugan, S. Kaushik, and S. Saravanan, Green synthesis of gold nanoparticles by using peltophorum pterocarpum flower extracts. Nano Biomed Eng, 2016, 8(4): 213-218.

[9] S. Palmal, N.R. Jana, Gold nanoclusters with enhanced tunable fluorescence as bioimaging probes. Wiley Interdiscip Rev Nanomed Nanobio-technol, 2014, 6(1): 102-110.

[10] D. Hu, Z. Sheng, S. Fang, et al., Folate receptor-targeting gold nanoclusters as fluorescence enzyme mimetic nanoprobes for tumor molecular colocalization diagnosis. Theranostics, 2014, 4(2): 142-153.

[11] X. Yang, L. Gan, L. Han, et al., Facile preparation of chiral penicillamine protected gold nanoclusters and their applications in cell imaging. Chem Commun, 2013,
49(23): 2302-2304.

[12] L. Shang, N. Azadfar, F. Stockmar, et al., One-pot synthesis of near-infrared fluorescent gold clusters for cellular fluorescence lifetime imaging. Small, 2011, 7(18): 2614-2620.

[13] D. Hu, Z. Sheng, P. Zhang, et al., Hybrid gold-gadolinium nanoclusters for tumor-targeted NIRF/CT/MRI triplemodal imaging in vivo. Nanoscale, 2013, 5(4): 16241628.

[14] C. Sun, H. Yang, Y. Yuan, et al., Controlling assembly of paired gold clusters within apoferritin nanoreactor for in vivo kidney targeting and biomedical imaging. $J \mathrm{Am}$ Chem Soc, 2011, 133(22): 8617-8624.

[15] E. Boisselier, D. Astruc, Gold nanoparticles in nanomedicine: Preparations, imaging, diagnostics, therapies and toxicity. Chem Soc Rev, 2009, 38(6): 17591782.

[16] P. Ghosh, G. Han, M. De, et al., Gold nanoparticles in delivery applications. Adv Drug Del Rev, 2008, 60(11): 1307-1315

[17] C.K. Kim, P. Ghosh, C. Pagliuca, et al., Entrapment of hydrophobic drugs in nanoparticle monolayers with efficient release into cancer cells. J Am Chem Soc, 2009, 131(4): 1360-1361.

[18] S. Roux, B. Garcia, J.L. Bridot, et al., Synthesis, characterization of dihydrolipoic acid capped gold nanoparticles, and functionalization by the electroluminescent luminol. Langmuir, 2005, 21(6): 2526-2536.

[19] J. Wang, Y. Yue, G. Chen, et al., Protease-promoted drug delivery using peptide-functionalized gold nanoparticles. Soft Matter, 2011, 7(16): 7217-7222.

[20] C. Zhang, C. Li, Y. Liu, et al., Gold nanoclusters-based nanoprobes for simultaneous fluorescence imaging and targeted photodynamic therapy with superior penetration and retention behavior in tumors. Adv Funct Mater, 2015, 25(8): 1314-1325.

[21] Y. Ma, H. Fu, C. Zhang, et al., Chiral antioxidant-based gold nanoclusters reprogram DNA epigenetic patterns. Sci Rep, 2016, 6: 33436.

[22] M. Grzelczak, J. Perez-Juste, P. Mulvaney, et al., Shape control in gold nanoparticle synthesis. Chem Soc Rev, 2008. 37(9): 1783-1791.

[23] C. Zhou, M. Long, Y. Qin, et al., Luminescent gold nanoparticles with efficient renal clearance. Angew Chem Int Ed, 2011, 50(14): 3168-3172.

[24] A. M. Gamal-Eldeen, A.M. Mona, Abo-Zeid, et al., In vivo genotoxicity of gold nanorods in mouse bone marrow compared with cyclophosphamide. Nano Biomed Eng, 2016, 8(4): 306-314.

[25] C. Zhang, Z. Zhou, X. Zhi, et al., Insights into the distinguishing stress-induced cytotoxicity of chiral gold nanoclusters and the relationship with GSTP1. Theranostics, 2015, 5(2): 134-149.

[26] S. Hirn, M. Semmler-Behnke, C. Schleh, et al., Particle size-dependent and surface charge-dependent biodistribution of gold nanoparticles after intravenous administration. Eur J Pharm Biopharm, 2011, 77(3): 407416.

[27] C.A. Simpson, K.J. Salleng, D.E. Cliffel, et al., In vivo toxicity, biodistribution, and clearance of glutathionecoated gold nanoparticles. Nanomed Nanotechnol Biol Med, 2013, 9(2): 257-263.

[28] A. Prodi, F.L. Filon, Nano-scaled particles and fibres occupational exposure assessment: an integrated approach from air sampling to skin and surface contamination. Nano Biomed. Eng, 2016, 8(2): 91-104.

[29] P. Huang, J. Lin, S.J. Wang, et al., Photosensitizerconjugated silica-coated gold nanoclusters for fluorescence imaging-guided photodynamic therapy. Biomaterials, 2013, 34(19): 4643-4654. 
[30] C.L. Zhang, Z.J. Zhou, Q.R. Qian, et al., Glutathionecapped fluorescent gold nanoclusters for dual-modal fluorescence/X-ray computed tomography imaging. $J$ Mater Chem B, 2013, 1(38): 5045-5053.

[31] D. Cui, G. Jin, T. Gao, et al., Characterization of brcaa1 and its novel antigen epitope identification. Cancer Epidem Biomar, 2004, 13(7): 1136-1145.

[32] V. Afanassiev, J. Troppmair, M. Schuler, et al., Production and characterization of monoclonal antibodies against human bad protein. Hybridoma, 1998, 17(4): 383-387.

[33] W. Kan, R. Jing, Q. Qirong, et al., BRCAA1 monoclonal antibody conjugated fluorescent magnetic nanoparticles for in vivo targeted magnetofluorescent imaging of gastric cancer. J Nanobiotecg, 2011, 9(1): 23-34.

[34] C. Wang, C. Bao, S. Liang, et al., HAI-178 antibodyconjugated fluorescent magnetic nanoparticles for targeted imaging and simultaneous therapy of gastric cancer. Nanoscale Res Lett, 2014. 9: 274.

[35] S.A. Adefegha, O.S. Omojokun, G. Oboh, Modulatory effect of protocatechuic acid on cadmium induced nephrotoxicity and hepatoxicity in rats in vivo. Springer Plus, 2015. 4: 619.

[36] N. Tang, Y. Zhang, Z. Liu, et al., Correlation analysis between four serum biomarkers of liver fibrosis and liver function in infants with cholestasis. Biomedical Reports, 2016, 5(1): 107-112.

[37] L.A. New, C.E. Martin, R.P. Scott, et al., Nephrin tyrosine phosphorylation is required to stabilize and restore podocyte foot process architecture. J. Am. Soc. Nephrol., 2016, 27(8): 2422-2435.

[38] H. Putaala, R. Soininen, P. Kilpeläinen, et al., The murine nephrin gene is specifically expressed in kidney, brain and pancreas: Inactivation of the gene leads to massive proteinuria and neonatal death. Hum Mol Genet, 2001, 10(1): $1-8$.

[39] C. Faul, K. Asanuma, E. Yanagida-Asanuma, et al., Actin up: Regulation of podocyte structure and function by components of the actin cytoskeleton. Trends Cell Biol, 2007, 17(9): 428-437.

[40] N.V. Chevtchik, M. Fedecostante, J. Jansen, et al., Upscaling of a living membrane for bioartificial kidney device. Eur J Pharmacol, 2016, 790: 28-35.
[41] L. Shen, C.R. Weber, D.R. Raleigh, et al., Tight junction pore and leak pathways: A dynamic duo. Annu Rev Physiol, 2011, 73: 283-309.

[42] Y. Xia, W. Li, C.M. Cobley, et al., Gold nanocages: From synthesis to theranostic applications. Acc Chem Res, 2011, 44(10): 914-924.

[43] M.C. Daniel, D. Astruc, Gold nanoparticles: Assembly, supramolecular chemistry, quantum-size-related properties, and applications toward biology, catalysis, and nanotechnology. Chem Rev, 2004, 104(1): 293-346.

[44] M. E. Stewart, C. R. Anderton, L.B. Thompson, et al., Nanostructured plasmonic sensors. Chem Rev, 2008, 108(2): 494-521.

[45] A. R. Tao, S. Habas, P. Yang, Shape control of colloidal metal nanocrystals. Small, 2008, 4(3): 310-325.

[46] A. Nel, T. Xia, L. Madler, et al., Toxic potential of materials at the nano level. Science, 2006, 311: 622-627.

[47] K. Khanra, S. Panja, I. Choudhuri, et al., Bactericidal and cytotoxic properties of silver nanoparticle synthesized from root extract of asparagus racemosus. Nano Biomed. Eng., 2016, 8(1): 39-46.

[48] H. S. Choi, B. I. Ipe, P. Misra, et al., Tissue- and organselective biodistribution of nir fluorescent quantum dots. Nano Lett, 2009, 9(6): 2354-2359.

[49] J. Chen, B. Wiley, Z.Y. Li, et al., Gold nanocages: Engineering their structure for biomedical applications. Adv Mater, 2005, 17(18): 2255-2261.

[50] X. Zhang, J. Yang, S. Song, et al., Passing through the renal clearance barrier: Toward ultrasmall sizes with stable ligands for potential clinical applications. Int $J$ Nanomed, 2014, 9: 2069-2072.

Copyright@ 2017 Kunlu Liu, Haixia Li, Yajun Wu, Chunlei Zhang, Duanyun Si, Quansheng Li, Sa Zhang, Jiye Yin, Yuanyuan Liu, Wensheng Qu, Junhua Wu, Yingge Zhang, Ruibin Su, and Yuxia Wang. This is an open-access article distributed under the terms of the Creative Commons Attribution License, which permits unrestricted use, distribution, and reproduction in any medium, provided the original author and source are credited. 